\title{
BMJ Open Intensive versus standard blood pressure control in type 2 diabetes: a restricted mean survival time analysis of a randomised controlled trial
}

\author{
Sandra Shi (1) , ${ }^{1}$ Natalia Gouskova, ${ }^{1}$ Mehdi Najafzadeh, ${ }^{2}$ Lee-Jen Wei, ${ }^{3}$ \\ Dae Hyun $\mathrm{Kim}^{1,2,4}$
}

To cite: Shi S, Gouskova N, Najafzadeh M, et al. Intensive versus standard blood pressure control in type 2 diabetes: a restricted mean survival time analysis of a randomised controlled trial. BMJ Open 2021;11:e050335. doi:10.1136/ bmjopen-2021-050335

- Prepublication history for this paper is available online. To view these files, please visit the journal online (http://dx.doi. org/10.1136/bmjopen-2021 050335).

Received 17 February 2021 Accepted 18 August 2021

Check for updates

(C) Author(s) (or their employer(s)) 2021. Re-use permitted under CC BY-NC. No commercial re-use. See rights and permissions. Published by BMJ.

${ }^{1}$ Hinda and Arthur Marcus Institute for Aging Research, Hebrew SeniorLife, Boston,

Massachusetts, USA

${ }^{2}$ Division of

Pharmacoepidemiology and Phamacoeconomics, Brigham and Women's Hospital, Boston, Massachusetts, USA

${ }^{3}$ Harvard University T H Chan School of Public Health, Boston, Massachusetts, USA

${ }^{4}$ Beth Israel Deaconess Medical Center, Boston, Massachusetts, USA

Correspondence to

Dr Dae Hyun Kim;

daehyunkim@hsl.harvard.edu

\section{ABSTRACT}

Background Restricted mean survival time analysis offers an intuitive and robust summary of treatment effect compared with HRs.

Objective To examine the effect of intensive versus standard blood pressure (BP) control on death or cardiovascular events in type 2 diabetes.

Design Secondary analysis of the Action to Control Cardiovascular Risk in Diabetes Blood Pressure trial. Setting 77 sites in the USA and Canada.

Participants 4733 adults with type 2 diabetes at high risk for cardiovascular events.

Interventions Systolic BP target $<120 \mathrm{~mm} \mathrm{Hg}(\mathrm{n}=2371)$ versus $<140 \mathrm{~mm} \mathrm{Hg}(\mathrm{n}=2362)$.

Measurements Composite endpoint of death, non-fatal myocardial infarction or non-fatal stroke.

Results The mean event-free survival time over 5 years (1825 days) was similar between intensive and standard BP control (1716 vs 1714 days; mean difference, 1.3 (95\% $\mathrm{Cl}-18.1$ to 20.7) days). However, intensive BP treatment was more beneficial for those assigned to standard glycaemic control (1725 vs 1697 days; mean difference, 28.1 (95\% Cl 0.4 to 55.9 ) days), but not for those assigned to intensive glycaemic control (1706 vs 1731 days; mean difference, $-25.2(95 \% \mathrm{Cl}-52.3$ to 1.9$)$ days) ( $\mathrm{p}=0.008$ for interaction). In subgroup analysis, the mean event-free survival time difference between intensive and standard BP treatment was $-76.0(95 \% \mathrm{Cl}-131.8$ to -20.3$)$ days for those with cognitive impairment and $21.8(95 \% \mathrm{Cl}-24.0$ to 67.5) days for those with normal cognitive function $(p=0.008$ for interaction). The effect was not different by age, sex and baseline cardiovascular disease status. Conclusions Intensive BP treatment may reduce death and cardiovascular events among patients with type 2 diabetes receiving standard glycaemic treatment and without cognitive impairment.

Trial registration number NCT00000620; Post-results.

\section{INTRODUCTION}

The benefits of intensive blood pressure (BP) control over standard BP control on major cardiovascular events have been demonstrated for non-diabetic adults with hypertension and elevated cardiovascular risk. ${ }^{12}$ Current guidelines from the Eighth Joint National
Strengths and limitations of this study

- Restricted mean survival time is intuitive and robust to modelling assumptions.

- This post-hoc analysis of a randomized controlled trial precludes definitive causal conclusions.

- Limited power for smaller subgroups, including cognitive function subgroup analysis.

Committee recommend treating to a systolic BP (SBP) target of $<150 \mathrm{~mm} \mathrm{Hg}$ for adults $>60$ years. ${ }^{13}$ However, this has been controversial given recent studies supporting cardiovascular benefit of intensive BP treatment in older adults, including the Systolic Blood Pressure Intervention Trial (SPRINT). Thus 2017 American Heart Association and American College of Cardiology guidelines recommend an SBP goal of $<130 \mathrm{~mm} \mathrm{Hg}$. Importantly, although SPRINT did not enrol older adults with diabetes, patients with diabetes are at particularly high risk of cardiovascular morbidity and mortality. Thus, the risks and benefits of intensive BP treatment among older adults with diabetes remains an area of clinical uncertainty.

The landmark study in patients with type 2 diabetes, the Action to Control Cardiovascular Risk in Diabetes (ACCORD) trial, failed to demonstrate benefits of intensive versus standard $\mathrm{BP}$ control in major cardiovascular event reduction ( $\mathrm{HR}, 0.88 ; 95 \% \mathrm{CI}$ 0.73 to 1.06$).{ }^{4}$ As the original $2 \times 2$ randomised study design also found intensive glycaemic control to be associated with increased risk of major cardiovascular events, secondary analyses were pursued to examine subgroup effects. Post-hoc analyses suggested a benefit of intensive BP control among those who were assigned to standard glycaemic control ( $\mathrm{p}$ for interaction $=0.08$ ),$^{4}$ these results were not statistically significant. However, 
these inconclusive results may have stemmed from lack of power due to the small number of events within these subgroups.

Additionally, a recent post-hoc analysis of SPRINT among participants 80 years or older suggests that the effect of intensive BP control may be greater in those without cognitive impairment (HR, $0.49 ; 95 \%$ CI 0.33 to 0.73 ) than in those with cognitive impairment at baseline (HR, 1.04; 95\% CI 0.65 to 1.66 ) ( $\mathrm{p}$ for interaction $=0.01){ }^{6}$ Thus, it remains unclear whether intensive BP control benefits (1) patients with type 2 diabetes and (2) whether there is variation by baseline cognitive function.

Restricted mean survival time (RMST) represents an event-free survival time in a time window. Graphically it represents the area under the survival curve. As a measure of treatment effect, RMST is more robust to modelling assumptions than traditional HRs from Cox regression. ${ }^{78}$ Because the difference in RMST between two treatments means a gain or loss in the mean event-free time due to the treatment compared with a control interpretation may be more intuitive. Such a time-based summary may complement HRs by helping patients perceive the magnitude of the treatment effect. This may be particularly useful for shared decision-making for older adults in whom the expected benefit needs to be considered within a time period (eg, remaining life expectancy).

We conducted a post-hoc analysis of ACCORD-Blood Pressure (ACCORD-BP) trial data using RMST analysis to provide a more robust and clinically interpretable metric of treatment efficacy. Here, we present results using RMST of intensive versus standard BP control in patients with type 2 diabetes and across subgroups, including pretreatment cognitive function.

\section{METHODS}

\section{Study design and population}

ACCORD was a multicentre randomised clinical trial, which enrolled 10251 participants with type 2 diabetes. ${ }^{49}$ Details of study design and patient recruitment have been previously published with the main findings of the study. ${ }^{10} 11$ Briefly, participants were randomly assigned to receive either standard (haemoglobin Alc target $7 \%-7.9 \%$ ) or intensive glycaemic control (haemoglobin A1c target $<6.0 \%$ ). In a 2-by-2 factorial design, 4733 participants were also randomly assigned to either standard (target SBP $<120 \mathrm{~mm} \mathrm{Hg}$ ) or intensive BP control (target $\mathrm{SBP}<140 \mathrm{~mm} \mathrm{Hg}$ ).

Recruitment took place in two non-contiguous periods, first during a 'vanguard phase' from January 2001 to June $2001 \quad(n=491)$, and the majority from January 2003 through October 2005 during the main trial. Participants were recruited from 77 clinical sites across the USA and Canada, using a diverse array of strategies. ${ }^{12}$ Participants were eligible if they (1) had type 2 diabetes; (2) had a haemoglobin A1c $\geq 7.5 \%$ and (3) were 40 years or older with clinical or subclinical cardiovascular disease (CVD) or 55 years or older with at least two additional risk factors for CVD. In order to take part in the BP trial, participants also had to have an SBP of $130-180 \mathrm{~mm} \mathrm{Hg}$ taking $<3$ BP medications. Exclusion criteria included body mass index $>45 \mathrm{~kg} / \mathrm{m}^{2}$, serum creatinine $>1.5 \mathrm{mg} / \mathrm{dL}$ and other serious illness.

\section{Participant consent}

All ACCORD participants provided informed consent at the time of trial enrolment. This manuscript was prepared using ACCORD Research Materials obtained from the National Health Lung, and Blood Institute (NHLBI) Biologic Specimen and Data Repository Information Coordinating Center and does not necessarily reflect the opinions or views of the ACCORD or the NHLBI.

\section{Interventions}

Participants were non-blinded to their BP or glycaemic control targets. BP was monitored during regular visits as part of the trial, assessed every 2-4 months and as needed. Medications were added and adjusted to achieve BP targets. Treatment algorithms have been previously published. ${ }^{10}$ After 1 year, mean SBP was $119.3 \mathrm{~mm} \mathrm{Hg}$ in the intensive BP control group and $133.5 \mathrm{~mm} \mathrm{Hg}$ in the standard group. ${ }^{4}$ Similarly, glycaemic control was achieved via glucose lowering medications, provided from a study-supervised formulary. ${ }^{11}$ Regimens were individualised at investigator discretion based on treatment assignment (haemoglobin A1c goal $<6.0 \%$ vs $7.0 \%-7.9 \%$ ). The median haemoglobin A1c levels at 1 year were $6.4 \%$ and $7.5 \%$, respectively, which were maintained for study duration. ${ }^{13}$

\section{Outcomes}

The primary outcome was a composite endpoint of nonfatal myocardial infarction, non-fatal stroke or death. The events were adjudicated by a central committee which was blinded to treatment assignment, with details of each prespecified endpoint available in the original publication. ${ }^{4}$

\section{Measurements}

High risk of CVD was operationalised as those with two or more CVD risk factors, including those with body mass index $>32 \mathrm{~kg} / \mathrm{m}^{2}$, current smokers, on BP-lowering medications or lipid-lowering medications. Subclinical CVD was defined as those with evidence of high likelihood of CVD, including the presence of microalbuminuria, reduced ankle brachial index, left ventricular hypertrophy or carotid stenosis. Existing CVD was defined as presence of previous myocardial infarction or stroke. Cognitive function was measured using mini-mental state examination (MMSE) as part of ACCORD Memory in Diabetes (MIND) substudy started 34 months after the start of ACCORD. ${ }^{14} 15$ Thus, baseline MMSE scores were only available for 1439 participants. 'Because there were a small number of participants $(n=148)$ with an MMSE score less than 24 points (conventional cutpoint used for dementia screening), we defined cognitive impairment as an MMSE score $\leq 27$ points'. 
Table 1 Characteristics of study population by blood pressure treatment strategy

Blood pressure treatment strategy

\begin{tabular}{|c|c|c|c|}
\hline Characteristics & Standard & Intensive & $P$ value \\
\hline Sample size & 2371 & 2362 & \\
\hline Age, years, mean (SD) & $62.8 \pm 6.8$ & $62.7 \pm 6.6$ & 0.86 \\
\hline \multicolumn{4}{|c|}{ Cardiovascular risk category } \\
\hline Existing CVD & 790 (33.3) & $805(34.1)$ & 0.85 \\
\hline 2 or more CVD risk factors & $1142(48.2)$ & $1127(47.7)$ & \\
\hline \multicolumn{4}{|c|}{ Glycaemic treatment strategy } \\
\hline Standard & 1178 (49.7) & $1184(50.1)$ & 0.76 \\
\hline Intensive & $1193(50.3)$ & 1178 (49.9) & \\
\hline \multicolumn{4}{|l|}{ Cognitive function $^{\star}$} \\
\hline Impaired (MMSE <28) & $304(43.8)$ & $311(41.7)$ & \\
\hline
\end{tabular}

*MMSE was only available in participants of Action to Control Cardiovascular Risk in Diabetes Memory in Diabetes study in the standard group $(n=694)$ and the intensive group $(n=745)$.

CVD, cardiovascular disease; MMSE, mini-mental status examination.

\section{Statistical analysis}

Baseline characteristics were compared between the intensive and standard BP control groups using a t-test for continuous variables and $\chi^{2}$ test for categorical variables. The effect of intensive BP control on time to the primary endpoint was assessed by comparing RMST between the intensive and standard BP control groups over a time horizon of 5 years (or 1825 days). This time window was chosen for RMST analysis because only a small proportion of participants remained at risk after 5 years (the mean follow-up duration was 4.7 years for the primary outcome). ${ }^{4}$ We also calculated restricted mean time lost (RMTL) due to CVD events (non-fatal myocardial infarction, non-fatal stroke or cardiovascular death) or due to non-cardiovascular death (competing risk). ${ }^{16}$ We conducted a regression analysis of RMST using pseudo-observations approach with the identity link. ${ }^{17}$ The models included the treatment group as the main

Table 2 RMST of blood pressure treatment strategy, stratified by glycaemic treatment strategy

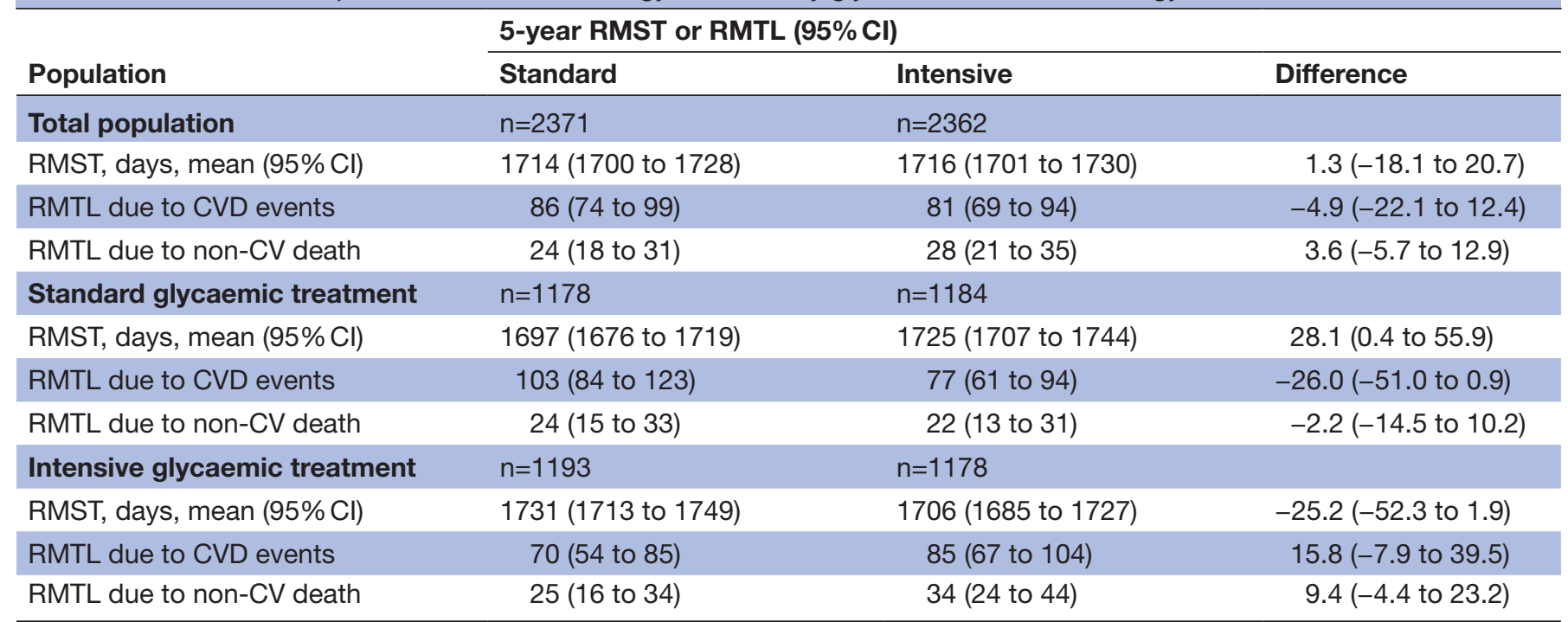

The interaction between blood pressure treatment strategy and glycaemic treatment strategy on RMST was statistically significant $(p=0.008)$.

CVD, cardiovascular disease; RMST, restricted mean survival time; RMTL, restricted mean time lost. 
A

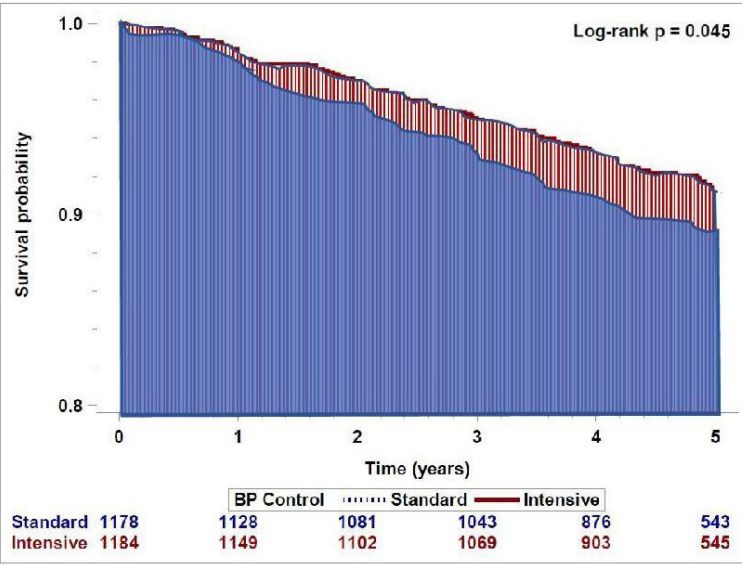

B

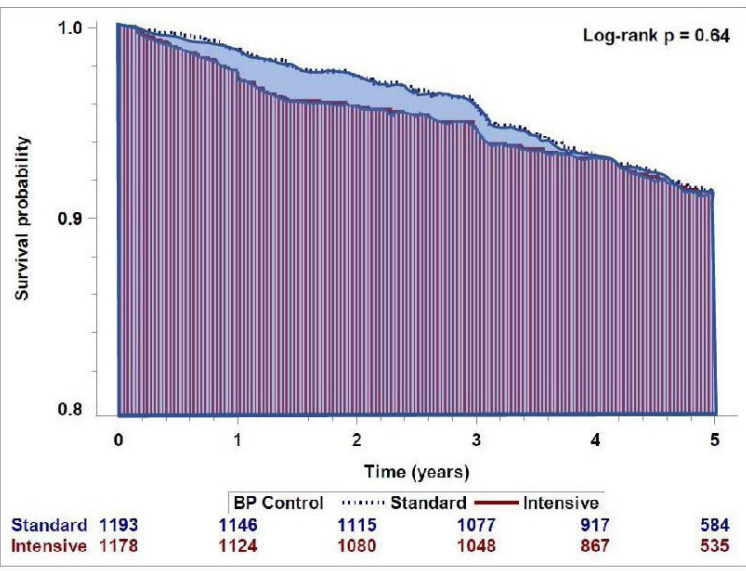

Figure 1 Kaplan-Meier curves by BP control group and glycaemic control group. (A) Standard glycaemic treatment; (B) intensive glycaemic treatment. The area shaded under the curves represent restricted mean survival time, blue line for standard BP control and red line for intensive BP control. Note that in (B) with the intensive glycaemic treatment cohort there is a violation of the proportional hazard assumption. BP, blood pressure.

predictor of interest and were adjusted for the recruitment network to mimic the original trial analysis. We reported adjusted least square means for RMST for each treatment group and their difference along with the $95 \% \mathrm{CI}$. We repeated this analysis in the overall population, as well as stratifying by glycaemic control strategy (standard vs intensive) and baseline characteristics, including age, sex, cognitive impairment and CVD risk category (existing CVD, subclinical CVD or high-risk group). In addition, we plotted the Kaplan-Meier curves and conducted the log-rank tests overall and by glycaemic control group using the data from the first 5 years to match the RMST analysis. Statistical analysis was performed using R V.3.6.0 (package cmprsk) and SAS V.9.4 (SAS Institute) software.

\section{Role of funding sources}

The National Institutes of Health provided funding for this investigator-initiated trial but did not participate in study design; collection, management, analysis or interpretation of data; writing of publications or the decision to submit any report for publication. The sponsor did not have authority over any of these activities.

\section{Patient and public involvement}

Patients and/or the public were not involved in the design, or conduct, or reporting, or dissemination plans of this research.

\section{RESULTS}

\section{Baseline characteristics}

Baseline characteristics were balanced between the standard $(\mathrm{n}=2371)$ and intensive $(\mathrm{n}=2362)$ BP control groups (table 1$)$, including the mean (SD) age (62.8 (6.8) vs $62.7(6.6))$, the number (\%) of females $(1130(47.7 \%)$ vs $1128(47.8 \%)$ ) and CVD risk groups (two or more CVD risk factors: 1142 (48.2) vs 1127 (47.7); subclinical CVD: 439 (18.5) vs 430 (18.2); existing CVD: 790 (33.3) vs 805 (34.1)). Among a subset with MMSE scores $(n=1439)$, mean MMSE score (SD) was 27.2 (2.6) points in the standard BP control group and 27.3 (2.7) points in the intensive $\mathrm{BP}$ control group.

\section{Effect of blood pressure control strategy on cardiovascular event-free time}

Overall, the 5-year RMST was similar between intensive BP control group (1716 (95\% CI 1701 to 1730 ) days) and standard BP control group (1714 (95\% CI 1700 to 1728 ) days), with a mean difference of 1.3 (95\% CI -18.1 to 20.7) days (table 2). RMTL due to cardiovascular and non-cardiovascular deaths were also similar. However, intensive BP control had different effects depending on the glycaemic control strategy ( $p$ for interaction $=0.008$ ).

Among those who were assigned to standard glycaemic control strategy, intensive BP control group showed higher event-free survival than standard control group (figure 1A, log-rank test $\mathrm{p}=0.045$ ). The 5 -year RMST was 1697 (95\% CI 1676 to 1719) days for the standard group, and 1725 (95\% CI 1707 to 1744) days for the intensive group; with a mean gain of 28.1 (95\% CI 0.4 to 55.9) days (table 2). That is, for patients who received standard glycaemic control, intensive BP control strategy delayed first major cardiovascular event by, on average, 28.1 days (ranging from 0.4 to 55.9 days) over 5 years compared with standard BP control strategy. Time lost due to noncardiovascular death was not different between standard or intensive BP control (-2.2 days (95\% CI -14.5 to 10.2)).

By contrast, among those who were assigned to the intensive glycaemic control strategy, intensive BP control group initially showed worse event-free survival than standard control group, but two survival curves merged over time (figure 1B, log-rank test $\mathrm{p}=0.64$ ). The 5-year RMST was 1731 (95\% CI 1713 to 1749) days for the standard group with 1706 (95\% CI 1685 to 1727) days for the intensive group, and a mean loss of 25.2 (95\% CI -52.3 to 1.9) days in the intensive group. In other words, for patients who received intensive glycaemic control, intensive BP control strategy shortened time to first major cardiovascular event by, on average, 
Age

.$<70$ years

$1726(1711,1740) 1725(1710,1740)$

. $70+$ years

$1653(1612,1695) 1666(1626,1706)$

Sex

. Male

$1706(1687,1725) 1715(1696,1735)$

Female

$1724(1704,1743) 1716(1695,1737)$

CVD Risk Status

. Existing CVD

$1670(1643,1698) 1656(1626,1685)$

. High likelihood of CVD $1674(1638,1711) 1720(1688,1752)$

$2+$ CVD risk factors

$1760(1745,1776) \quad 1757(1741,1773)$

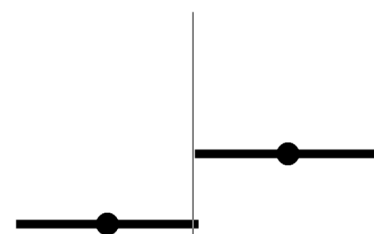

$28(0,56)$

$-26(-53,2) \quad<0.01$

$-1(-21,20)$

$13(-45,70) \quad 0.67$

Cognitive function

. Normal

$1699(1635,1762) 1721(1660,1782)$

$\longrightarrow$

$22(-23,68)$

Impaired

$1737(1677,1796) \quad 1662(1591,1733)$

-100 (standard better) 0 (intensive better) 100

Figure 2 RMST of blood pressure treatment strategy by subgroups. Differences in RMST, in days, for standard versus intensive blood pressure control, by subgroups. CVD, cardiovascular disease; MMSE, mini-mental status examination; RMST, restricted mean survival time.

25.2days (ranging from 52.3 days shorter to 1.9 days longer) over 5 years compared with standard BP control strategy.

\section{Subgroup analyses}

Among participants with cognitive impairment, RMST for the intensive BP control group was $76.0(95 \%$ CI -131.8 to -20.3) days shorter than that of the standard BP control group, suggesting potential deleterious effects of intensive $\mathrm{BP}$ control. This contrasts with a mean RMST gain of 21.8days (95\% CI -24.0 to 67.5 ) among those with normal cognitive function at baseline (figure 2). No statistically significant variation by age, sex and CVD risk category was observed.

\section{DISCUSSION}

In this post-hoc analysis of ACCORD trial we examined the effect of intensive versus standard BP control on death or cardiovascular events in type 2 diabetes, using RMST over a 5-year timeframe. Overall, we found that mean event-free survival time over 5 years was similar between intensive and standard BP control. Importantly however, our results provide evidence of heterogeneity of treatment effects. There was more event-free time with intensive BP control among those receiving standard glycaemic control. However, those receiving intensive glycaemic control did not benefit from intensive BP control. Furthermore, those with cognitive impairment at baseline had fewer event-free days with intensive BP control than standard BP control.

The main findings of ACCORD concluded that there was increased mortality with intensive glycaemic control compared with standard glycaemic control. ${ }^{18}$ ACCORD-BP failed to show the benefit of intensive BP control on major cardiovascular events compared with standard BP control. ${ }^{4}$ Our study extends these findings, by demonstrating that while dual intensive therapy was detrimental, intensive BP control alone in fact conferred a modest benefit in time without major cardiovascular event. This is more consistent with another large randomised controlled trial among older adults, the SPRINT, ${ }^{1}$ which found that intensive BP control reduced major cardiovascular events and allcause mortality (HR $0.75(0.64-0.89))$. Indeed, when 
analysed with RMST at roughly 4 years participants had a statistically significant 13.8 days more major adverse cardiovascular event-free days compared with standard BP control. ${ }^{19}$ However, SPRINT excluded those with diabetes, while ACCORD exclusively enrolled those with diabetes. Our findings highlight possible heterogeneity of treatment effects, suggesting that some diabetics may still benefit from intensive BP control.

A previous study using data from the ACCORD-BP trial reported significantly lower risk of major cardiovascular events in the intensive BP control group among patients received standard glycaemic control (HR, 0.71; 95\% CI 0.56 to 0.90 ), over a mean follow-up of 4.5 years, and there was a significant interaction between BP control and glycaemic control strategies $(p=0.02) .{ }^{20}$ However, in this study as well as other similar post-hoc analyses the Kaplan-Meier survival curves are crossing among patients who received intensive glycaemic control, ${ }^{21}$ which is a violation of the proportional hazards assumption. Some other post-hoc analyses also considered variable effects by kidney disease. ${ }^{22}$ Our work here corroborates these findings, while highlighting the advantages of the RMST approach, as RMST-based statistical tests remain appropriate even if proportional hazards assumptions are violated.

Previous literature in RMST has demonstrated how reanalysis of previously published trials allows for intuitive interpretation of treatment efficacy. ${ }^{79}{ }^{23}$ Although a minimum clinically important difference in RMST has not been established, this in fact allows for patients to personally determine how many days would be meaningful. Thus, it may offer easier application into shared decision-making. For example, for patients with life expectancy of less than 1 year, a quantified number of days gained may help to place advantages or disadvantages in better perspective. Additional research should focus on the utility of RMST-based communication of evidence in improving providers' ability to explain treatment effect to patients as well as patients' understanding of the treatment effect.

An important finding to highlight is that those with cognitive impairment at baseline did not benefit from intensive BP control, and in fact had less event-free time. As mentioned previously, cognitive testing was not incorporated into the main protocol of ACCORD and was introduced subsequently as part of a substudy, ACCORD MIND. ${ }^{14}{ }^{15}$ Of note, the ACCORD MIND study did not observe significant differences between cognitive outcomes, which taken into consideration with the overall increased mortality among participants in the intensive glycaemic control group, did not favour use of intensive glycaemic control. Further analyses failed to find benefit for intensive BP control with regard to cognitive function as well. ${ }^{24}$ Although the association between BP control and the incidence of dementia has been extensively explored, with evidence of supporting the use of antihypertensive medications to lower dementia risk, there has been less evidence to support intensive BP control for prevention of major cardiovascular events among those who have pre-existing cognitive impairment. Furthermore, these patients are often excluded from clinical trials. ${ }^{25-27}$ Our findings suggesting differential effects and possible harm, should prompt further investigation by cognitive status.

Our study has important limitations. As a secondary analysis, this finding should be interpreted with caution and cannot be considered confirmatory. We did not have time to event data to conduct RMST analysis for adverse events. In the original publication, serious adverse events occurred more often in the intensive BP control group than standard group (77 (3.3\%) vs $30(1.7 \%), \mathrm{p}<0.001) .{ }^{4}$ RMST analysis depends on the choice of time horizon, which should be prespecified. Using an earlier time point (or shorter time horizon) may result in less of a difference. We chose 5 years as this was the time window specified in the originally published analyses, ${ }^{4}$ in which most events occurred. This gives an adequate time frame in which benefit from $\mathrm{BP}$ reduction is expected to be observed (12-18 months). ${ }^{28}$

In conclusion, in our analysis of intensive versus standard BP control among patients with type 2 diabetes, there was no significant difference in event-free time between standard and intensive BP control. However, we found a significant event-free time gained of 28 days over 5 years for those who received intensive BP control and standard glycaemic control, which was not observed in the intensive glycaemic control. Finally, those with cognitive impairment had shorter event-free time with intensive BP control. These results support the presence of treatment heterogeneity and highlight the need for personalised decision-making in this diverse population.

\section{Twitter Sandra Shi @shisadoctor}

Contributors Obtaining data: DHK. Analysis: NG. Interpretation of findings: SS, NG, DHK, L-JW, MN. Drafting of manuscript: SS, NG, DHK. All authors read and approved the final manuscript.

Funding This study was funded by R21AG060227 from the National Institute on Ageing (NIA) to DHK.

Competing interests None declared.

Patient consent for publication Not required.

Ethics approval The Hebrew SeniorLife Institutional Review Board approved this study.

Provenance and peer review Not commissioned; externally peer reviewed.

Data availability statement Data are available in a public, open access repository. This manuscript was prepared using ACCORD Research Materials obtained from the National Health Lung, and Blood Institute (NHLBI) Biologic Specimen and Data Repository Information Coordinating Center and does not necessarily reflect the opinions or views of the ACCORD or the NHLBI.

Open access This is an open access article distributed in accordance with the Creative Commons Attribution Non Commercial (CC BY-NC 4.0) license, which permits others to distribute, remix, adapt, build upon this work noncommercially, and license their derivative works on different terms, provided the original work is properly cited, appropriate credit is given, any changes made indicated, and the use is non-commercial. See: http://creativecommons.org/ licenses/by-nc/4.0/. 
ORCID iD

Sandra Shi http://orcid.org/0000-0001-6801-5602

\section{REFERENCES}

1 SPRINT Research Group, Wright JT, Williamson JD, et al. A randomized trial of intensive versus standard blood-pressure control. N Engl J Med 2015;373:2103-16.

2 Beckett NS, Peters R, Fletcher AE, et al. Treatment of hypertension in patients 80 years of age or older. N Engl J Med 2008;358:1887-98.

3 James PA, Oparil S, Carter BL, et al. 2014 evidence-based guideline for the management of high blood pressure in adults. JAMA 2014;311:507.

4 ACCORD Study Group, Cushman WC, Evans GW, et al. Effects of intensive blood-pressure control in type 2 diabetes mellitus. $N$ Engl J Med 2010;362:1575-85.

5 Margolis KL, O'Connor PJ, Morgan TM, et al. Outcomes of combined cardiovascular risk factor management strategies in type 2 diabetes: the Accord randomized trial. Diabetes Care 2014;37:1721-8.

6 Pajewski NM, Berlowitz DR, Bress AP, et al. Intensive vs Standard Blood Pressure Control in Adults 80 Years or Older: A Secondary Analysis of the Systolic Blood Pressure Intervention Trial. J Am Geriatr Soc 2020;68:496-504.

7 Kloecker DE, Davies MJ, Khunti K, et al. Uses and limitations of the restricted mean survival time: illustrative examples from cardiovascular outcomes and mortality trials in type 2 diabetes. Ann Intern Med 2020;172:541-52.

8 Tian L, Zhao L, Wei LJ. Predicting the restricted mean event time with the subject's baseline covariates in survival analysis. Biostatistics 2014;15:222-33.

9 Action to Control Cardiovascular Risk in Diabetes Study Group, Gerstein HC, Miller ME, et al. Effects of intensive glucose lowering in type 2 diabetes. N Engl J Med 2008;358:2545-59.

10 Cushman WC, Grimm RH, Cutler JA, et al. Rationale and design for the blood pressure intervention of the action to control cardiovascular risk in diabetes (accord) trial. Am J Cardiol 2007;99:S44-55.

11 Gerstein HC, Riddle MC, Kendall DM, et al. Glycemia treatment strategies in the action to control cardiovascular risk in diabetes (accord) trial. Am J Cardiol 2007;99:S34-43.

12 Kingry C, Bastien A, Booth G, et al. Recruitment strategies in the action to control cardiovascular risk in diabetes (accord) trial. Am J Cardiol 2007;99:S68-79.

13 Dluhy RG, McMahon GT. Intensive glycemic control in the ACCORD and advance trials. N Engl J Med 2008;358:2630-3.

14 Launer LJ, Miller ME, Williamson JD, et al. Effects of intensive glucose lowering on brain structure and function in people with type
2 diabetes (accord mind): a randomised open-label substudy. Lancet Neurol 2011;10:969-77.

15 Murray AM, Hsu F-C, Williamson JD, et al. ACCORDION mind: results of the observational extension of the Accord mind randomised trial. Diabetologia 2017;60:69-80.

16 Zhao L, Tian L, Claggett B, et al. Estimating treatment effect with clinical interpretation from a comparative clinical trial with an end point subject to competing risks. JAMA Cardiol 2018;3:357-8.

17 Anderson PK, Hansen MG, Klein J. Regression analysis of restricted mean survival time based on pseudo-observations for competing risks data. Commun Stat - Theory Methods 2018;47:5614-25.

18 ACCORD Study Group, Gerstein HC, Miller ME, et al. Long-Term effects of intensive glucose lowering on cardiovascular outcomes. $N$ Engl J Med 2011;364:818-28.

19 Krishnaswami A, Peterson ED, Kim DH, et al. Efficacy and safety of intensive blood pressure therapy using restricted mean survival Time-Insights from the sprint trial. Am J Med 2020;133:e369-70.

20 Tsujimoto T, Kajio H. Benefits of intensive blood pressure treatment in patients with type 2 diabetes mellitus receiving standard but not intensive glycemic control. Hypertension 2018;72:323-30.

21 Beddhu S, Chertow GM, Greene T, et al. Effects of intensive systolic blood pressure lowering on cardiovascular events and mortality in patients with type 2 diabetes mellitus on standard glycemic control and in those without diabetes mellitus: reconciling results from accord bp and sprint. J Am Heart Assoc 2018;7:e009326.

22 Aggarwal R, Petrie B, Bala W, et al. Mortality outcomes with intensive blood pressure targets in chronic kidney disease patients. Hypertension 2019;73:1275-82.

$23 \mathrm{Kim} \mathrm{DH}$, Uno H, Wei L-J. Restricted mean survival time as a measure to interpret clinical trial results. JAMA Cardiol 2017;2:1179-80.

24 Williamson JD, Launer LJ, Bryan RN, et al. Cognitive function and brain structure in persons with type 2 diabetes mellitus after intensive lowering of blood pressure and lipid levels: a randomized clinical trial. JAMA Intern Med 2014;174:324-33.

25 Lee PY, Alexander KP, Hammill BG, et al. Representation of elderly persons and women in published randomized trials of acute coronary syndromes. JAMA 2001;286:708-13.

26 Sardar MR, Badri M, Prince CT, et al. Underrepresentation of women, elderly patients, and racial minorities in the randomized trials used for cardiovascular guidelines. JAMA Intern Med 2014;174:1868-70.

27 Taylor JS, DeMers SM, Vig EK, et al. The disappearing subject: exclusion of people with cognitive impairment and dementia from geriatrics research. J Am Geriatr Soc 2012;60:413-9.

28 Ickowicz E. Guiding principles for the care of older adults with multimorbidity: an approach for clinicians: American geriatrics Society expert panel on the care of older adults with multimorbidity. $J$ Am Geriatr Soc 2012;60:E1-25. 